\title{
A Test for the Presence of Covariance Between Time-Uncertain Series of Data with Application to the Dongge Cave Speleothem and Atmospheric Radiocarbon Records
}

\section{Citation}

Haam, Eddie, and Peter Huybers. 2010. A Test for the Presence of Covariance Between TimeUncertain Series of Data with Application to the Dongge Cave Speleothem and Atmospheric Radiocarbon Records. Paleoceanography 25, no. 2: PA2209.

\section{Published Version}

doi:10.1029/2008PA001713

\section{Permanent link}

http://nrs.harvard.edu/urn-3:HUL.InstRepos:13041036

\section{Terms of Use}

This article was downloaded from Harvard University's DASH repository, and is made available under the terms and conditions applicable to Other Posted Material, as set forth at http:// nrs.harvard.edu/urn-3:HUL.InstRepos:dash.current.terms-of-use\#LAA

\section{Share Your Story}

The Harvard community has made this article openly available.

Please share how this access benefits you. Submit a story.

\section{Accessibility}




\title{
A test for the presence of covariance between time-uncertain series of data with application to the Dongge Cave speleothem and atmospheric radiocarbon records
}

\author{
Eddie Haam ${ }^{1}$ and Peter Huybers ${ }^{2}$ \\ Received 29 October 2008; revised 11 March 2009; accepted 14 August 2009; published 2 June 2010.
}

[1] Statistical measures of the relationships between time series are generally altered by the presence of errors in timing, i.e., when applied to time-uncertain series. For example, the covariance sampled between two time series which in truth covary will generally be decreased by errors in timing. Most previous work on this subject has sought to maximize some goodness of fit between time-uncertain series either heuristically or through more quantitative methods. However, there is a danger that unrelated records can be made to appear to covary by time adjustment. Here we propose a statistical test for the presence of covariance between time-uncertain series wherein the probability of obtaining a maximum covariance from randomly realized time-uncertain series is assessed using the theory of order statistics. The results of this analytical method provide insight into the influence of timing errors upon covariance and are shown to be consistent with results derived from a Monte Carlo procedure. We apply this methodology to evaluate the covariance between a time-uncertain stalagmite record and atmospheric radiocarbon during the Holocene and find, contradictory to previous interpretation, that there is insignificant covariance between the two at the $95 \%$ confidence level.

Citation: Haam, E., and P. Huybers (2010), A test for the presence of covariance between time-uncertain series of data with application to the Dongge Cave speleothem and atmospheric radiocarbon records, Paleoceanography, 25, PA2209, doi:10.1029/2008PA001713.

\section{Introduction}

[2] To understand the Earth's climate, we must understand the interrelations between its specific geographical areas which, in the case of paleoclimatology, can be profitably undertaken from an empirical perspective. However, assessment of the interrelation between separate paleoclimate records is hindered by uncertainties in the absolute and relative timing of these climate records. It thus appears necessary, if we are to take full advantage of the paleoclimate record, to develop techniques that are capable of evaluating the relationship between series of data whose times are uncertain, i.e., time-uncertain series.

[3] At some level, the time assigned to a discrete measurement is always uncertain, but when such uncertainties are minimal, they can largely be neglected. Many simple and useful statistical methods are available to test for relationship between time series [e.g., Casella and Berger, 2002], but when time uncertainties are large, as described in more detail later, they can substantially influence the statistics used to evaluate time series. For example, errors in time can change the mean value, variance, and autocorrelation structure of continuous data, as well as series of

\footnotetext{
${ }^{1}$ Applied Mathematics, Harvard University, Cambridge, Massachusetts, USA.

${ }^{2}$ Department of Earth and Planetary Sciences, Harvard University, Cambridge, Massachusetts, USA.
}

Copyright 2010 by the American Geophysical Union. 0883-8305/10/2008PA001713 discrete data once interpolated to some regular or other spacing. Furthermore, errors in relative timing will generally strongly influence the sampled relationship between timeuncertain series.

[4] Time uncertainty is often addressed as an optimization problem wherein some relationship between time-uncertain series is to be maximized through time adjustments. For example, various algorithms have been proposed to maximize the cross correlation, to maximize covariance, or to minimize the sum of the residual of squared difference between records within certain constraints [e.g., Macleod and Sadler, 1995; Huang et al., 2001; Lisiecki and Lisiecki, 2002; Huybers and Wunsch, 2004; Tomasi et al., 2004], while others have sought to maximize coherence [Clemens, 2005], variance explained by Empirical Orthogonal Functions [Clark et al., 2007], and the relationship between Empirical Mode Decompositions of time-uncertain records [Solé et al., 2007]. Mudelsee [2001] and Delmotte et al. [2004] also studied phase relationships between two paleoclimate timeuncertain series. Many studies have also employed visual "wiggle matching" between time-uncertain series [Little et al., 1997; Schulz et al., 1998; Peterson et al., 2000; Wang et al., 2001; Hendy et al., 2002; Stott et al., 2002; Oppo et al., 2003; Pahnke et al., 2003; Genty et al., 2003]. Similar problems exist in other fields. For example, speech recognition software needs to cope with fast, slow, or uneven speech [e.g., Huang et al., 2001]. Airline flight scheduling must cope with uncertain arrival and departure times [e.g., Barnhart and Talluri, 1997]. The rate at which proteins produced by gene sequences is not consistent, resulting in time-uncertain gene expression serial data [e.g., Aach and Church, 2001]. Here 
too, the problem is generally treated as one of optimization within the time constraints.

[5] Visual and automated wiggle matching has provided useful insight regarding the interrelations of the climate system and the mechanisms behind such interrelations. Indeed, in some cases the correspondence between records is obvious upon inspection and expected on physical grounds, e.g., the $\delta^{18} \mathrm{O}$ of the ice measured in nearby Greenland ice cores. However, the relationship between most paleoclimate records is not beyond debate, particularly if significance is to be judged by individual pattern recognition skills [Clark and Thompson, 1979; Crowley, 1999; Wunsch, 2006, 2008]. Randomly generated signals which are unrelated can exhibit high covariance after adjustment within time uncertainties [Wunsch, 2003], and an objective measure to ascertain when observed covariances are unlikely to result from chance alone appears useful.

[6] Various statistical methods have been used to measure the likelihood of achieving maximum matching of timeuncertain series. For example, Anstey [1964] described various correlation techniques, whereas Clark and Thompson [1979] used a stretching function to align time-uncertain series and were able to report approximate confidence regions. Later, Clark [1989] designed a test wherein two time series are repeatedly randomly repartitioned into two groups. The maximum covariance found between these repartitioned groups was then used to assess the significance of the maximum covariance between the original two time series. However, these tests [Clark and Thompson, 1979; Clark, 1989] used the null hypothesis that the sequences were in fact equivalent, making it difficult to evaluate the significance of a match between records that only partially covary. Gordon and Buckland [1996] instead generated random series through permutation of the differences between consecutive elements of the sequence and employed a null hypothesis of no covariance between records, thus permitting testing of partial relationships between records. However, this approach did not fully account for the autocorrelation structure of the time series, which we find strongly influence the test results, and relied solely upon a Monte Carlo version of the test.

[7] Analytical representation of the statistical significance of covariances has been derived in the context of gene and protein sequence alignment [e.g., Levitt and Gerstein, 1998; Comet et al., 1999; Karlin and Altschul, 1990]. These results depend upon the discrete nature of the data and use extreme value theory, which is applicable in the case of very large numbers of degrees of freedom but not to the relatively short time series data focused on here.

[8] In this paper we derive the significance of a maximum covariance between time-uncertain series, under some limiting assumptions, using the theory of order statistics. We provide both a theoretical derivation of the distribution of maximum covariance as well as a Monte Carlo treatment. The theoretical derivation provides insight into the test while the Monte Carlo method is more practical to implement when the permitted combinations of the time model are large. It is worth noting that determination of statistical significance is distinct from the larger goal of ascertaining physical significance but that we expect the former to aid in realizing the latter.

\section{Design of the Test}

[9] In order to test for a relationship between the timeuncertain series, we need to choose a suitable null and alternative hypotheses, test statistic, and methodology for estimating the distribution of these hypotheses. Below we outline the specific choices that are made, and the rationale behind these decisions, but note that many variations upon this test procedure are possible, and which in certain contexts may be more advantageous. We refer to this statistical test as the Maximum Covariance between Time uncertain Series Test, MCTEST.

\subsection{Null and Alternative Hypotheses}

[10] Following Gordon and Buckland [1996], the null hypothesis that we will seek to reject is that two timeuncertain series do not covary. There are many possible alternate hypotheses depending on which data are being considered, the choice of which will influence the power of the test, i.e., the ability to distinguish between the null and alternate hypotheses. As the covariance assumed in the alternate hypothesis approaches zero, the power of the test also approaches zero. We choose perhaps the simplest alternate hypothesis, that the time-uncertain series covary perfectly and which then maximizes the statistical power.

\subsection{Test Statistic}

[11] Covariance is used to measure the similarity between time-uncertain series because it is amendable to analytical treatment within the context of order statistics. However, the method presented here is, in principle, extendable to more complicated measures of the relationship between records, including coherence and Pearson's correlation coefficient.

[12] We consider several means of measuring the covariance between time-uncertain records and distinguish between them according to their ability to differentiate between the null and alternative hypothesis. Our initial attempt at assessing covariance, which we ultimately abandoned, was to sample all covariances admissible given the time uncertainty and test whether the null hypothesis of a zero mean distribution could be rejected. However, we found that the average value was often indistinguishable from zero when the time uncertainty was large, even for highly covarying records, suggesting a low power of the test. Furthermore, it was difficult to estimate the effective degrees of freedom in the distribution of covariance. A variant was to test for the skewness of the correlation coefficient distribution. David [1938] showed that the distribution of the sample correlation coefficients is symmetric when the population correlation coefficient is zero, and skewed if the population correlation coefficient is nonzero, but again the degrees of freedom were unclear and the apparent power of the test was low.

[13] In keeping with previous approaches [Anstey, 1964; Clark and Thompson, 1979; Clark, 1989; Gordon and 
Buckland, 1996], we select to test the significance of the covariance between time-uncertain series based upon the maximum covariance found among all permitted time realization. This avoids the difficulty of estimating the number of degrees of freedom among all possible realizations because we assess only the single realization of the maximum covariance. Furthermore, the focus on the maximum relationship avoids the large pools of noncovarying realizations which develop when time uncertainty is large, leading to a higher power of the test. While estimation of the maximum covariance between time-uncertain series can be accomplished efficiently using, for example, dynamic programming [Tomasi et al., 2004], we employ an exhaustive search of a finite combination of times which the data series is permitted to take on because our theoretical derivation of the test depends upon assessment of all possible realizations and because this ensures identification of the actual maximum, within the selected group of time realizations.

\subsection{Distribution of the Null and Alternative Hypotheses}

[14] Of the various possibilities for representing the distribution of covariance under the null hypothesis, we choose those which serve to increase the analytical tractability of the problem and which preserve as much of the original data structure as possible.

[15] In order to ensure that the null hypothesis of zero covariance between time-uncertain series is met, it is necessary to modify or replace one or both of the observed timeuncertain series. We choose to replace one time-uncertain series with a randomly realized time series having a similar autoregressive behavior and to retain the other time-uncertain series in its original form. It is convenient to retain that time series which is more difficult to model using an autoregressive model. The retention of a fixed time series and its comparison against a random autoregressive realization means that the resulting distribution of the covariance will be normally distributed, as opposed to the more complicated chi-square distribution which would result from replacing both time-uncertain series with random models.

[16] In general, both of the time series under consideration will be time uncertain. It would be possible to individually model the time uncertainty in both records, but we note that the expected covariance between time-uncertain series is only a function of the relative timing errors without specification of which series that timing error belongs to, e.g., $\operatorname{cov}\left(y\left(t+\epsilon_{y}\right), x\left(t+\epsilon_{x}\right)\right)=\operatorname{cov}\left(y\left(t+\epsilon_{y}-\epsilon_{x}\right), x(t)\right)$, at least up to issues involved with resampling of the records. For purposes of making the problem more tractable, we choose to assign all the relative time uncertainty between records to a single record, and represent the other as being time certain. To generate the null distribution, it is more convenient to replace the time series chosen as time certain with random series because time-certain series maintain a constant autocorrelation.

[17] Note that we will explicitly represent the autocorrelation associated with each time series. Previous techniques [Clark and Thompson, 1979; Clark, 1989; Gordon and Buckland, 1996] did not fully account for the autocorrelated nature of the time series. Consequently, those test results may be biased by "spurious correlation" because the degrees of freedom and associated statistical significance depend on the autocorrelation of the time series [e.g., Yule, 1926; Granger and Newbold, 1974]. Our method takes full account of the autocorrelation in time series and the influence this has on the estimation of statistical significance.

[18] The relative time uncertainties associated with each pair of records are represented as a piecewise linear function. The intersections of the linear pieces are assumed independent and take on discrete values that are uniformly probable. Such a simple model of the relative time uncertainty may not hold in detail for many records. For example, Rahmstorf [2003] and Buck and Blackwell [2004] discuss that time uncertainties are not necessarily independent, Stuiver et al. [1998] showed that the distribution of time uncertainties are generally nonuniform, and Blaauw et al. [2004] discuss the use of a piecewise nonlinear model. However, we are guided by the desire to fashion a test which is sufficiently simple as to be amendable to thorough evaluation. We later discuss methodologies for dealing with more nuanced distributions of time uncertainty. We also note that the assumption of a piecewise linear, independent, discrete, and uniform distribution of time uncertainty resembles what is often implicitly assumed in algorithms that seek to maximize the match between time-uncertain records [e.g., Lisiecki and Lisiecki, 2002; Huybers and Wunsch, 2004].

\subsection{Notation}

[19] It is useful to establish a notation to represent a timeuncertain series as the standard indexing against time is inadequate. We represent time-uncertain series as $y\left(n, t_{n}^{\prime}\right)$, where $n$ denotes a specific data point and $t_{n}^{\prime}$ represents the distribution of possible times associated with that $n$th point. We use the more conventional notation, $x(t)$, to represent a time-certain series. The null distribution can therefore be expressed as the probability distribution of the maximum covariance between $y\left(n, t_{n}^{\prime}\right)$ and $X(t)$, according to which the maximum covariance between $y\left(n, t_{n}^{\prime}\right)$ and $x(t)$ can be tested, and where we will define $X(t)$ as representing the collection of time series having the same autocovariance as $x(t)$.

\section{Analytical Derivation of the MCTEST}

[20] It is simple enough to determine the maximum covariance between time-uncertain series, especially if the problem is designed such that an exhaustive search is possible. The effort we make is instead to determine the significance of the maximum covariance through derivation of the distribution of the null hypothesis which the sample covariance will be assessed against. We consider three increasingly complicated versions of the MCTEST: (1.) $y(n=1$, $t_{n=1}^{\prime}=3,4$, or 5) having only one data point with three possible time assignments, each having equal probability; (2.) $y\left(n=1, . ., 5, t_{n=3}^{\prime}=3\right.$, 4 , or 5$)$ having 5 data points but whose times all linearly depend on the third time-uncertain data point; and (3.) a case with five data points and two uncertain times. From here, the statistical method is trivially extendable to arbitrarily large data sets (excepting issues related to computational resources) and we will apply it to a time series from Dongge Cave [Wang et al., 2005] 

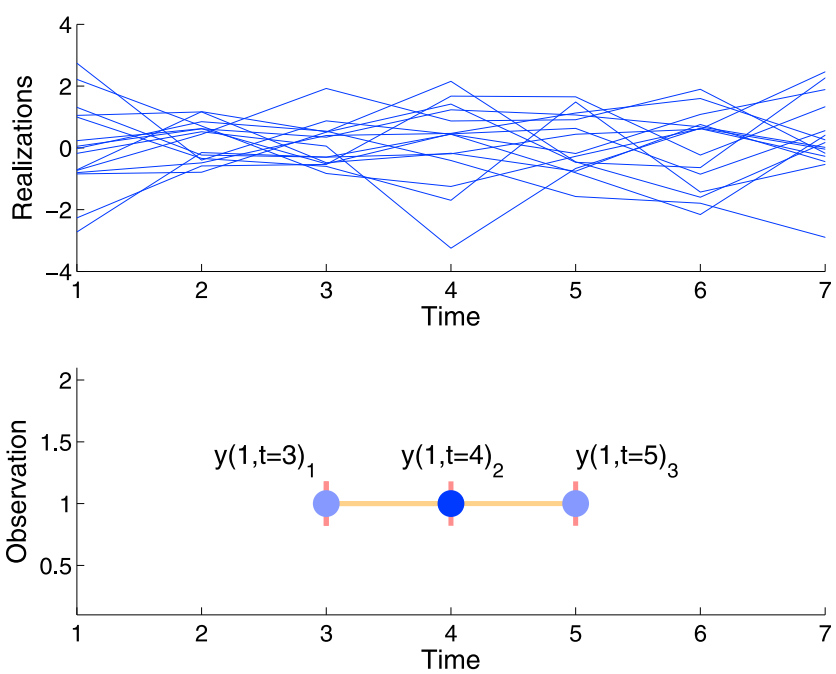

Figure 1. A sketch indicating the covariance between $X(t)$ and $y(1, t)$. (top) $X(t)$ is a random variable, and (bottom) $y\left(n, t_{n}^{\prime}\right)$ is one data point at $t=4$ with 1 unit time uncertainty. Since $y\left(n, t_{n}^{\prime}\right)$ has three possible locations at $t=3,4$, or 5 , there are three possible covariances with $X(t):[y(1,3)$. $X(3)],[y(1,4) \cdot X(4)]$, or $[y(1,5) \cdot X(5)]$. with 889 data points and 48 points with independent time uncertainty.

\subsection{Maximum Covariance Between White Noise and a Single Time-Uncertain Data Point}

[21] The covariance is a measure of the degree to which two time series vary in the same direction. Given two realizations of time series $x(t)$ and $y(t)$ with an expected means of zero, the covariance is $\operatorname{Cov}(x(t), y(t))=\frac{1}{N} \sum_{t=1}^{N} x(t) \cdot y(t)$. From this equation, it follows that the covariance between a standard normally distributed random variable, $X(t)$, and a realization of a time series, $y(t)$, is normally distributed. We assume expected value of $X$ equals to zero.

[22] Now consider the product of one data point, $y(n=1$, $t_{n=1}^{\prime}=3,4$ or, 5) $=1$, and a random process that is independent and normally distributed with zero mean and unity standard deviation, $X(t)=\varepsilon_{t}$ (see Figure 1). While a time series having but a single entry is somewhat odd, it permits illustration of the test in a maximally simple manner. Absent time uncertainty, the covariance is $y\left(n=1, t_{n=1}=4\right) \cdot X(t=4)$, which equates with the distribution of $X(t=4)$. With the time uncertainty, however, there exist three possible covariances: $\left[y\left(1, t_{1}=3\right) \cdot X(3)\right],\left[y\left(1, t_{1}=4\right) \cdot X(4)\right]$ and $\left[y\left(1, t_{1}=5\right) \cdot X(5)\right]$. The maximum covariance is thus $y\left(n=1, t^{\prime}\right)$ multiplied by the maximum of $X(3), X(4)$ and $X(5)$. The cumulative

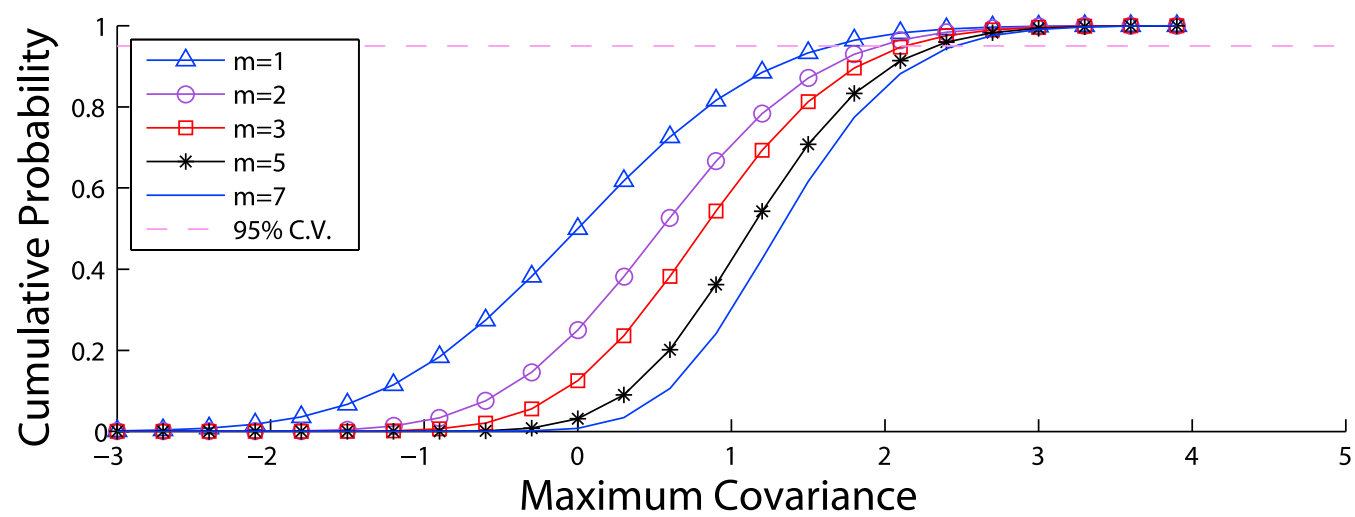

(a)

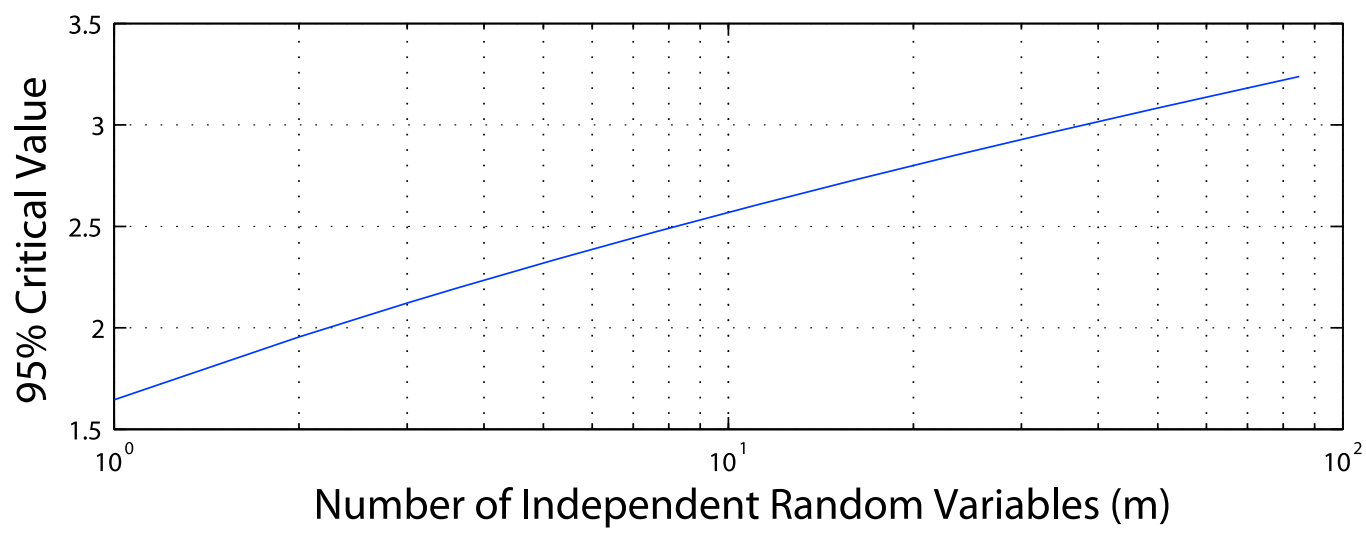

(b)

Figure 2. (a) The cumulative distribution of the maximum covariance of $m$ independent, normally distributed random variables. (b) The $95 \%$ critical value as a function of $m$. 


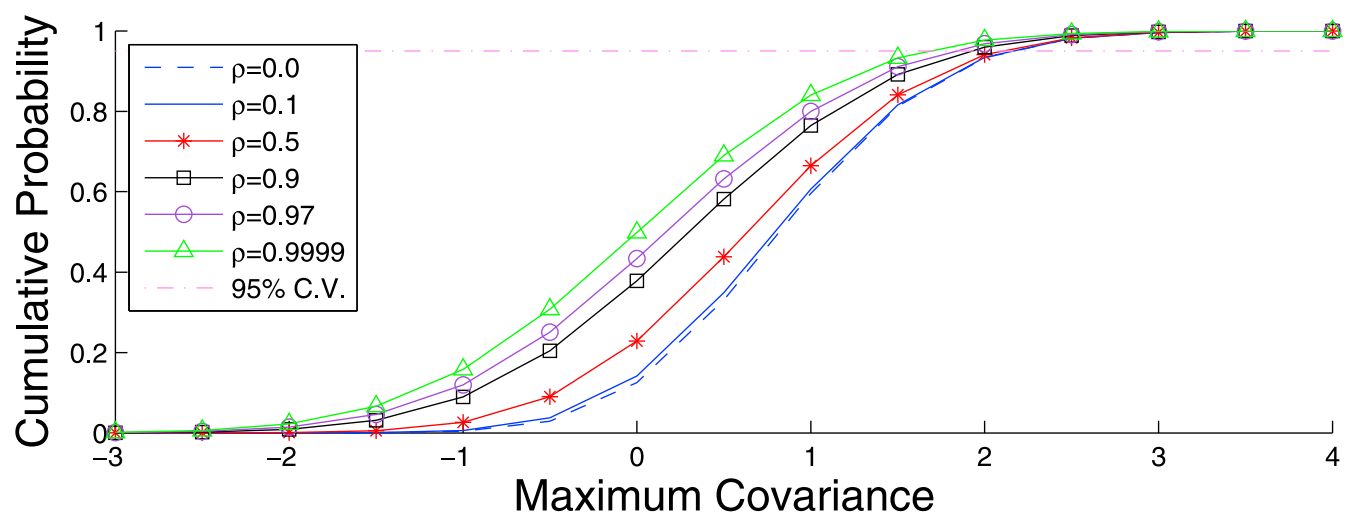

(a)

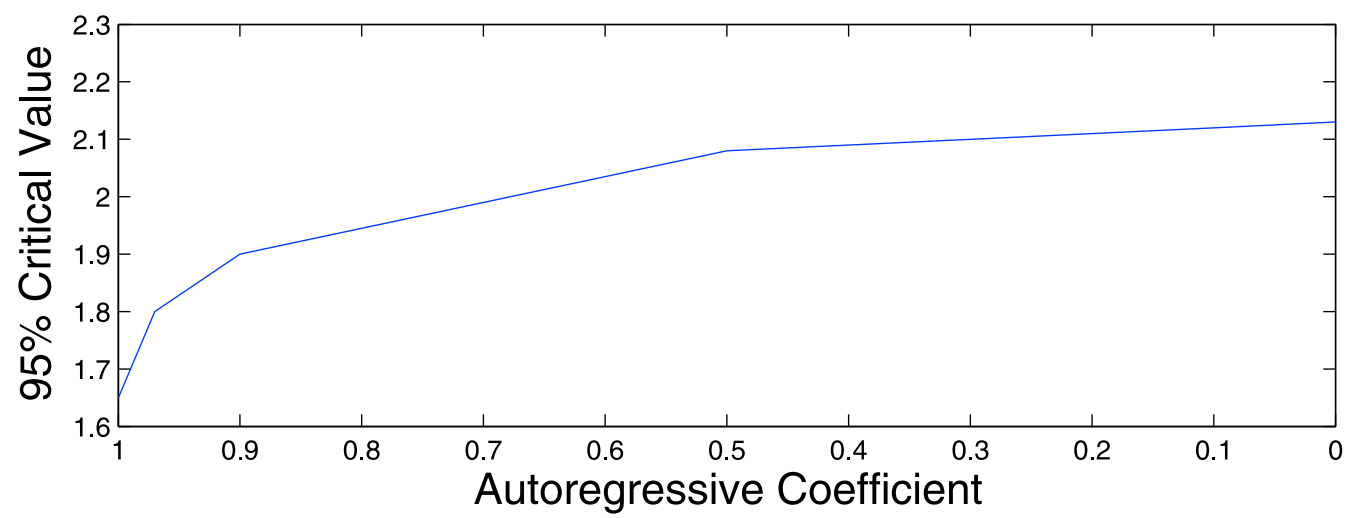

(b)

Figure 3. Similar to Figure 2 but now for the case of autocorrelated $x(t)$ represented using an autoregressive order one process with a varying coefficient, $\rho$. (a) The cumulative distribution of the maximum covariance when the covariance matrix is given by equation (3). (b) The $95 \%$ critical values increase as the autoregressive coefficient decreases.

distribution of the maximum of $X(3), X(4)$ and $X(5)$ is [David and Nagaraja, 2003]

$$
\begin{aligned}
F_{\max (X(3), X(4), X(5))} & =P[X(3) \leq c \text { and } X(4) \leq c \text { and } X(5) \leq c], \\
& =P[X(3) \leq c] \cdot P[X(4) \leq c] \cdot P[X(5) \leq c], \\
& =P[X \leq c]^{3}=F_{X}(c)^{3} .
\end{aligned}
$$

where the fact that $X(t)$ is independent and identically distributed has been used. $F_{X}(c)$ is the cumulative standard normal distribution and has the same mean and variance as $X(t)$. The cumulative distribution of maximum covariance is thus $y(n=1) \cdot F_{X}(c)^{3}$.

[23] If the number of discrete time points which $y(n=1)$ can take on is $m$, then the cumulative distribution of the maximum is $y(n=1) \cdot F_{X}(c)^{m}$, assuming each point is equally likely. As the time uncertainty increases, the bounds over which a maximum covariance can be searched for also increases, and both $m$ and the probability of obtaining higher covariance increases (Figure 2a). The analytical form of the $95 \%$ critical value as a function of $m$ is obtained by rearranging the equation from $F^{m}\left(c_{0.95}\right)=0.95$ to $F\left(c_{0.95}\right)=(0.95)^{1 / m}$ and then $c_{0.95}=F^{-1}\left(0.95^{1 / m}\right)$. As one would expect, the $95 \%$ critical value increases monotonically as $m$ increases (Figure 2b).

\subsection{Maximum Covariance Between Autocorrelated Noise and a Time-Uncertain Data Point}

[24] Next we consider the case when $X(t)$ is an autocorrelated random variable. The autocorrelation structure will differ according to the climate data but we can begin with a simple first-order autocorrelated random time series, $X(t)=\rho X(t-1)+\varepsilon_{t}^{\prime}$. The term $\varepsilon_{t}^{\prime}$ represents a white noise process with zero mean which is specified to have a variance of $\left(1-\rho^{2}\right)$ such that $X(t)$ has unit variance for $0<\rho<1$.

[25] Building from the previous example, the distribution of maximum covariance is still defined as $y(n=1)$. $\max (X(3), X(4), X(5))$. However, $X(t=3,4,5)$ are no longer independent, and we need to evaluate $P(X(3) \leq c$ and $X(4) \leq c$ and $X(5) \leq c)$ taking into account that $\operatorname{Cov}(X(3)$, $X(4))=\operatorname{Cov}(X(4), X(5))=\rho$, and that $\operatorname{Cov}(X(3), X(5))=\rho^{2}$. The expected autocorrelation can be defined as $\rho^{m}$, where $m$ is the number of time steps between two variables and the covariance between points is $\operatorname{Cov}\left(X\left(t_{1}\right), X\left(t_{2}\right)\right)=\rho^{\left|t_{1}-t_{2}\right|}$. Because $y(n=1) \cdot X(t)$ is normally distributed, the cumulative distribution of the maximum covariance is a multivariate normal integration,

$$
\begin{aligned}
& P(X(3) \leq c \text { and } X(4) \leq c \text { and } X(5) \leq c) \\
& \quad=\int_{-\infty}^{c} \int_{-\infty}^{c} \int_{-\infty}^{c} \frac{1}{(2 \pi)^{N / 2}|\Sigma|^{1 / 2}} e^{-\frac{1}{2}(\nu-\mu)^{T} \Sigma^{-1}(\nu-\mu)} d \nu
\end{aligned}
$$




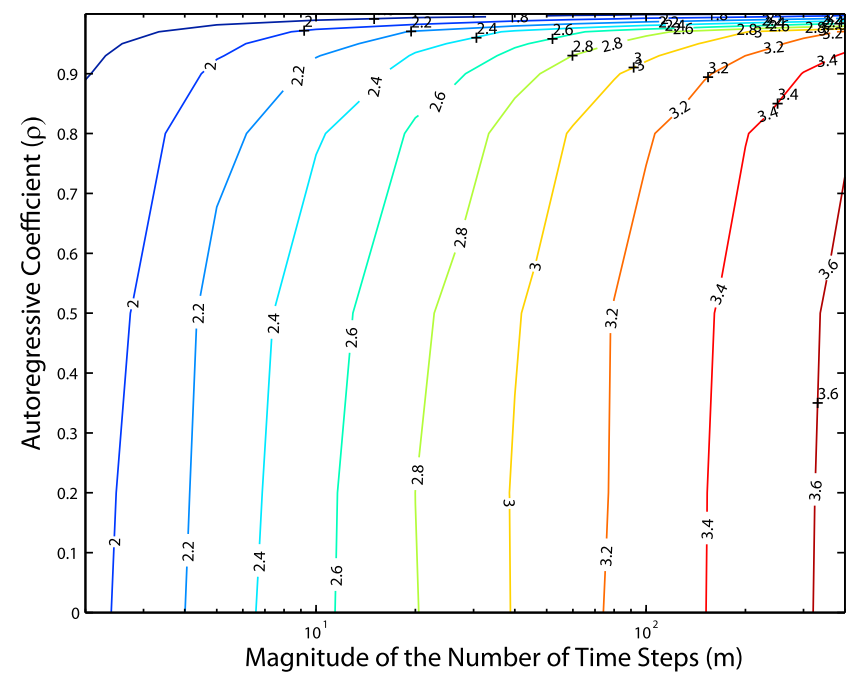

Figure 4. The $95 \%$ critical value as a function of the magnitude of the number of time steps, $m$, and the autoregressive order-one coefficient, $\rho$ (see equation (4)). The $95 \%$ critical value increases monotonically as $m$ increases because more realizations are possible and as $\rho$ decreases because the different realizations become more distinct.

where $\boldsymbol{\mu}$ is the vector of means of the multivariate normal and $\nu$ is integrated from $-\infty$ to $c$. The covariance matrix is

$$
\begin{aligned}
\Sigma & =\left(\frac{1}{N}\right)^{2} y(n=1)^{2} \\
& \cdot\left(\begin{array}{lll}
\operatorname{Cov}(X(3), X(3)) & \operatorname{Cov}(X(3), X(4)) & \operatorname{Cov}(X(3), X(5)) \\
\operatorname{Cov}(X(4), X(3)) & \operatorname{Cov}(X(4), X(4)) & \operatorname{Cov}(X(4), X(5)) \\
\operatorname{Cov}(X(5), X(3)) & \operatorname{Cov}(X(5), X(4)) & \operatorname{Cov}(X(5), X(5))
\end{array}\right) \\
& =\left(\frac{1}{N}\right)^{2} y(n=1)^{2}\left(\begin{array}{ccc}
1 & \rho & \rho^{2} \\
\rho & 1 & \rho \\
\rho^{2} & \rho & 1
\end{array}\right) .
\end{aligned}
$$

[26] As $\rho$ decreases, the off-diagonal terms approach zero, and the orthogonality of the covariance matrix increases. The number of distinct eigenvalues of the covariance matrix is indicative of the degrees of freedom in the problem. It follows that the mean value, skewness, and $95 \%$ critical value of the maximum covariance all increase with decreasing $\rho$ (see Figure 3). Oppositely, as $\rho$ increases toward one, the distribution approaches univariate normal. If $\rho=1, X(t)$ becomes nonstationary and the MCTEST is no longer applicable. Note that time adjustments that are small relative to the autocorrelation length scale will yield little change in the expected maximum covariance. We will call on this property later, when it becomes necessary to limit the number of realizations we integrate over.
[27] If the size of the time uncertainty increases, the size of the covariance matrix also increases:

$$
\Sigma=\left(\frac{1}{N}\right)^{2} y(n=1)^{2}\left(\begin{array}{ccccc}
1 & \rho & \rho^{2} & \cdots & \rho^{m} \\
\rho & 1 & \rho & \cdots & \rho^{m-1} \\
\vdots & \vdots & \vdots & \vdots & \vdots \\
\rho^{m} & \cdots & \rho^{2} & \rho & 1
\end{array}\right),
$$

where $m$ is now the magnitude of the number of time steps. Figure 4 shows how the $95 \%$ critical value increases as $m$ increases and $\rho$ decreases, consistent with the interpretation that these changes serve to increase the degrees of freedom represented in the covariance matrix.

\subsection{Distribution of the Maximum Covariance Between Autocorrelated Random Noise and a Time-Uncertain Series}

[28] Consider the case of a time series, $y\left(n, t_{n}^{\prime}\right)$, with five data points which has fixed times at the ends, $t_{1}^{\prime}=0 \pm 0$ and $t_{5}^{\prime}=7 \pm 0$, an uncertain time in the center, $t_{3}^{\prime}=4 \pm 1$, and two intervening data points whose time linearly depends on the center time (see Figure 5). We will call points with independent times, i.e., $y(n=1,3,5)$, Time Control Points (TCPs). As is commonly done in the construction of time models [e.g., Shaw, 1964], the times of non-TCPs are linearly interpolated for according to the neighboring TCPs. Thus, taking $t_{n}^{\circ}$ as the initial time estimate and $t_{n}^{\prime}$ as indicating the range of times that are permissible, we have the relationship

$$
\frac{\left(t_{3}^{\prime}-t_{2}^{\prime}\right)}{\left(t_{3}^{\prime}-t_{1}^{\prime}\right)}=\frac{\left(t_{3}^{\circ}-t_{2}^{\circ}\right)}{\left(t_{3}^{\circ}-t_{1}^{\circ}\right)}
$$
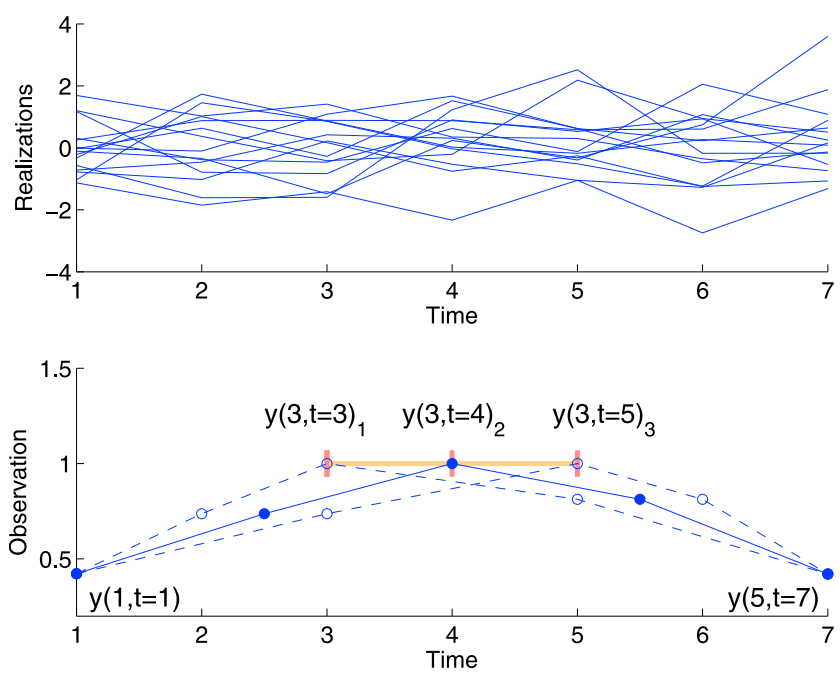

Figure 5. Similar to Figure 1 but for a more complicated $y\left(n, t_{n}^{\prime}\right) . X(t)$ is an autoregressive order one process, $X(t)=$ $\rho X(t-1)+\varepsilon_{t}^{\prime}$, where $\varepsilon^{\prime}{ }_{t}$ is white noise with variance of $(1-$ $\left.\rho^{2}\right)$. Here $y\left(n, t_{n}^{\prime}\right)$ has a TCP at $n=3$ with one unit time uncertainty and two fixed points at $n=1$ and $n=5$. The dotted line represents two other possible realizations of the time series. 


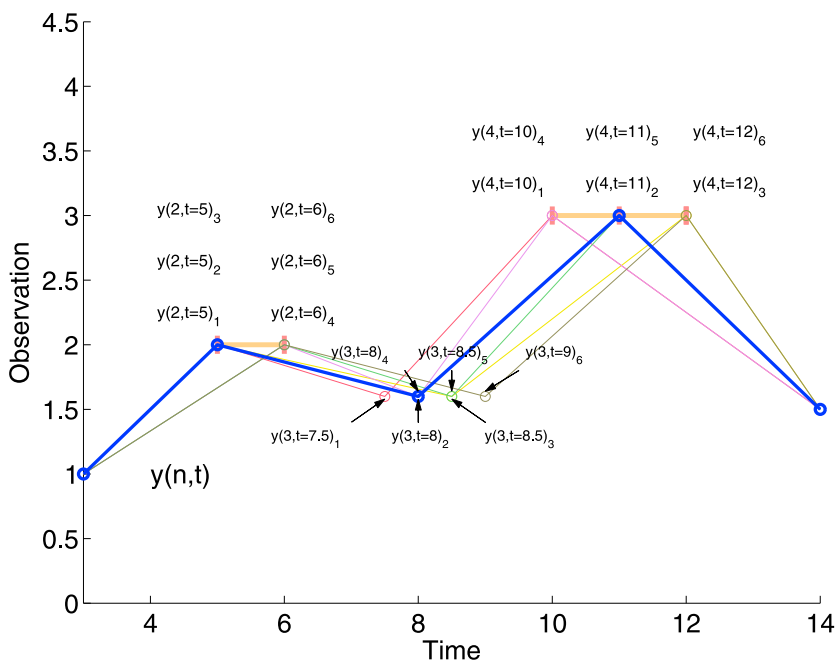

Figure 6. Similar to Figure 5 but for two TCPs. The TCPs at $n=1$ and $n=5$ are time certain, while the time at $n=2$ has one unit uncertainty to the +1 direction and the time at $n=4$ has an uncertainty of \pm 1 . The 6 possible time realizations are shown.

and that

$$
t_{2}^{\prime}=\frac{-\left(t_{3}^{\circ}-t_{2}^{\circ}\right)\left(t_{3}^{\prime}-t_{1}^{\prime}\right)}{\left(t_{3}^{\circ}-t_{1}^{\circ}\right)}+t_{3}^{\prime}
$$

The time of $t_{4}^{\prime}$ is determined analogously.

[29] There are three possible realizations of the timeuncertain series (Figure 5) which, using equation (5), lead to three possible covariances,

$$
\begin{aligned}
\mathrm{C}_{1}= & \frac{1}{N}[y(1,1.0) X(1.0)+y(2,2.0) X(2.0)+y(3,3.0) X(3.0) \\
& +y(4,5.0) X(5.0)+y(5,7.0) X(7.0)], \\
\mathrm{C}_{2}= & \frac{1}{N}[y(1,1.0) X(1.0)+y(2,2.5) X(2.5)+y(3,4.0) X(4.0) \\
& +y(4,5.5) X(5.5)+y(5,7.0) X(7.0)], \\
\mathrm{C}_{3}= & \frac{1}{N}[y(1,1.0) X(1.0)+y(2,3.0) X(3.0)+y(3,5.0) X(5.0) \\
& +y(4,6.0) X(6.0)+y(5,7.0) X(7.0)],
\end{aligned}
$$

where $X(2.5)$ and $X(5.5)$ are obtained by linear interpolation. Each of the covariances follow a normal distribution, and also covary amongst each other, so the distribution of the maximum must again be evaluated by solving a multivariate normal integration (equation (2)) with a covariance matrix,

$$
\Sigma=\left(\begin{array}{lll}
\operatorname{Cov}\left(C_{1}, C_{1}\right) & \operatorname{Cov}\left(C_{1}, C_{2}\right) & \operatorname{Cov}\left(C_{1}, C_{3}\right) \\
\operatorname{Cov}\left(C_{2}, C_{1}\right) & \operatorname{Cov}\left(C_{2}, C_{2}\right) & \operatorname{Cov}\left(C_{2}, C_{3}\right) \\
\operatorname{Cov}\left(C_{3}, C_{1}\right) & \operatorname{Cov}\left(C_{3}, C_{2}\right) & \operatorname{Cov}\left(C_{3}, C_{3}\right)
\end{array}\right)
$$

Each of the covariance terms can be evaluated using the following general form:

$$
\begin{aligned}
\sum_{i j} & =\operatorname{Cov}\left(C_{i}, C_{j}\right) \\
& =\frac{1}{N^{2}} \cdot \sum_{k=1}^{N} \sum_{l=1}^{N} \operatorname{Cov}\left[y\left(k, t_{k i}^{\prime}\right) X\left(t_{k i}^{\prime}\right), y\left(l, t_{l j}^{\prime}\right) X\left(t_{l j}^{\prime}\right)\right],
\end{aligned}
$$

where $t^{\prime}{ }_{k i}$ is the $i$ th time realization for data point $k$, and $t_{l j}^{\prime}$ is the $j$ th time realization for data point $l$.

\subsection{Multiple Independent Time Control Points}

[30] Finally, we consider a five point time series with two TCPs with nonzero timing uncertainty at $y\left(n=2, t_{2}^{\prime}=5\right.$ or 6$)$ and $y\left(n=4, t_{4}^{\prime}=11 \pm 1\right)$, one intervening point at $y\left(n=3, t_{3}^{\prime}\right)$, and fixed end points (Figure 6). There is essentially no difference with the foregoing example, but we include this to demonstrate how the method can be scaled to larger timeuncertain series. There are six possible realizations of the time series. The general form of the covariance for realization $i$ of the time series is

$$
\begin{aligned}
C_{i}= & \frac{1}{N}\left(y(1,3) X(3)+y\left(2, t_{2 i}^{\prime}\right) X\left(t_{2 i}^{\prime}\right)+y\left(3, t_{3 i}^{\prime}\right) X\left(t_{3 i}^{\prime}\right)\right. \\
& \left.+y\left(4, t_{4 i}^{\prime}\right) X\left(t_{4 i}^{\prime}\right)+y(5,14) X(14)\right),
\end{aligned}
$$

where $i=1, . .6$.

[31] The times $t_{2 i}^{\prime}$ and $t_{4 i}^{\prime}$ are the $i$ th realized time for corresponding TCPs, and $t_{3 i}^{\prime}$ is computed using the method indicated by equation (5). Again, because each of the covariances are normally distributed, the distribution of the maximum covariance is found by integrating a multivariate normal distribution. The covariance matrix is evaluated using equation (8).

\subsection{Extension}

[32] This method can readily be extended from the autoregressive order one process example shown here to higher order autoregressive random variables, for example, using the sample autocorrelation of climate proxy data. Specifically, if we let $W(l)$ be the sample autocorrelation of $x(t)$ at lag $l$, the covariance matrix can be evaluated as $\operatorname{Cov}(X(a), X(b))=$ $W(b-a)$. For time series with length $N$, it appears best to model the time series as an order $N$ autoregressive process, i.e., $\operatorname{AR}(N)$. In later sections, we introduce the Monte Carlo version of MCTEST, which does not require an analytical representation of the probability density function of the series and thus can be readily extended to nonnormally distributed series such as the bimodal distribution of $\delta^{18} \mathrm{O}$ found in GRIP during the last glacial [Wunsch, 2003]. However, we should note these extensions are applicable under the limitation that the time series are stationary, that the relative time uncertainties associated with each pair of records can be represented as a piecewise linear function, that the intersections of the linear pieces are independent and take on discrete values, and that each discrete value is uniformly probable. 


\subsection{Plausible Adjustments to Time Control Points}

[33] Note that the present description of the time model permits the largest time adjustments at the TCPs, with a linear interpolation of time between. However, such a linear interpolation only provides an approximate indication of the time uncertainty because, for example, sediment accumulation rates or stalagmite growth rates are not steady. Furthermore, TCPs are often chosen to coincide with stratigraphic boundaries or radiometric time estimates, but because of nonsteady changes in depth or length with time, it is typically the case that intervals furthest removed from time estimates are the most uncertain [Huybers and Wunsch, 2004]. In future work, it will be useful to account for a more complete model of the structure of time uncertainty in data series.

[34] It is possible that the uncertainty associated with two consecutive TCPs would lead them to imply too high an accumulation rate or even to transpose positions. The plausible range of accumulation rate variations needs to be considered on a case-by-case basis according to the type of data and its specific environment [e.g., Sadler, 1981]. Further, the autocorrelation could be modeled between TCPs. It is then possible to omit time model realizations which fall outside the bounds of plausibility by excluding these combinations from the covariance matrix.

\section{Numerical Integration of a Multivariate Normal Distribution}

[35] The distribution of maximum covariance is expressed in the form of a multivariate normal integral, whose integration in all but the simplest cases is computationally demanding. Deriving a closed form solution for such integrals is an ongoing research topic in the statistics community, but it is nonetheless possible to derive some general features of the distribution analytically. Gupta [1963] presented a numerical method of solving the multivariate normal integral for the special case of equicorrelated variates. David and Nagaraja [2003] and Tong [1990] also present analytical results for equicorrelated variables. However, for paleoclimate or geologic time series, it is unlikely for the covariance matrix to have equal covariances; the autocorrelation of $X(t)$ generally decays with increasing lag.

[36] Analytical solutions are also possible if the multivariate normal integrals run from $-\infty$ to zero. Moran [1968] derived the analytical form $P\left(X_{1} \geq 0, X_{2} \geq 0\right)$ for a bivariate normal integral, also known as Sheppard's [1897] formula, as well as the trivariate normal case $P\left(X_{1} \geq 0, X_{2} \geq 0\right.$, $\left.X_{3} \geq 0\right)$. For higher-order cases $P\left(X_{1} \geq 0, X_{2} \geq 0, . . X_{n} \geq 0\right)$, David [1953] showed how to evaluate the integral using recurrence relations. These formulas do not fully determine the distribution of maximum covariance, but they can be used to get the mean and variance of the distribution [Afonja, 1972].

[37] Extreme value theory shows that as the number of independent data (degrees of freedom) increases, the distribution of the maximum from a multivariate normal random variable approaches a Gumbel distribution [Kotz, 2000], whose standard form is $e^{-e^{-(x-\mu) / \delta}}$. Husler [1961] derived the asymptotic parameters of a Gumbel distribution when the number of independent data is very large $(\geq 1000)$. This approximation could be useful if the number of independent realizations is large, but numerical tests have shown that Husler's asymptotic formula has an appreciable bias when the degrees of freedom are less than $10^{3}$. Note that even long climate time series may, in practice, have fewer than $10^{3}$ degrees of freedom because of autocorrelation. Thus we expect that approximation using the Gumbel distribution will not be applicable for most climate data.

[38] The two seemingly most practical methods of evaluating the multivariate normal integral are by Genz's method [Genz, 1993] and by a Monte Carlo multivariate integration method. Genz's method transforms the multivariate normal integral into a simpler integral by decomposing the covariance matrix using the Cholesky decomposition so that numerical evaluation is possible. The Monte Carlo multivariate integration method also depends on a Cholesky decomposition of the covariance matrix, which is then used to multiply a vector of normally distributed univariate random numbers to create multivariate random numbers. The maximums of these random numbers are then selected and their distribution approximates the distribution of the underlying multivariate normal integral. Both of these methods yield consistent results, and we used the Monte Carlo multivariate integration method.

[39] In order to evaluate the Cholesky Decomposition, the covariance matrix has to be positive definite. Since many of the possible time series realizations will be highly correlated and the autocorrelation structure is arbitrary, the covariance matrix is not necessarily positive definite. We thus use a spectral decomposition to find a positive definite approximation to the covariance matrix by eliminating nonpositive eigenvalues [e.g., Anderson et al., 1999]. If the magnitude and proportion of the nonpositive eigenvalues are small, the spectral decomposition approximation is sufficiently accurate.

\section{Approximation}

[40] The number of possible realizations of time models associated with a time-uncertain series can be computed as $\Pi_{i=1}^{N} m_{i}$, where $m_{i}$ is the number of discrete time uncertainties for each TCP, $i$, and $N$ is the total number of TCPs. If, for example, each of 10 TCPs has five admissible times, the size of the covariance matrix is $5^{10}$ by $5^{10}$. Evaluation of such a large covariance matrix would require prohibitive computing resources. Thus, prior to applying the MCTEST, it is first useful to introduce two approximations for reducing the size of the covariance matrix that is apt to result from a paleoclimate time-uncertain series of even moderate length.

\subsection{Approximation by Elimination of Highly Correlated Realizations}

[41] We conjecture that if two realizations of a single timeuncertain series are highly correlated, one of the realizations can be removed from the covariance matrix without significantly changing the results of the MCTEST. That is, we propose to reduce the set of all possible realizations to a smaller set of more distinct realizations. We could search for highly correlated pairs of all possible realizations, eliminate one of the highly correlated pairs, and continue in this fashion until all pairs satisfy some distinctness criteria. However, this 


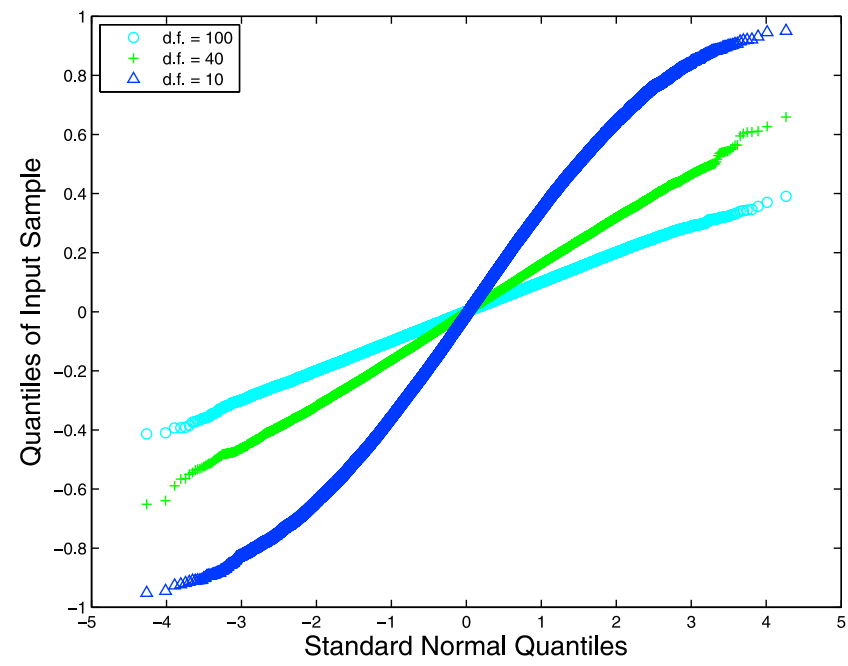

Figure 7. Quantile-quantile plot of correlation coefficients having different degrees of freedom (d.f.). We observe approximate normality for degrees of freedom greater than 40 .

process also requires substantial computing resources because of the number of realizations which must be compared.

[42] A more practical approach is to exclude realizations which are expected to be highly correlated, without actually generating and comparing them. For example, eliminating realizations which differ from one another by only one time unit may have little influence on the results, depending on the degree of autocorrelation.

\subsection{Monte Carlo Simulation of MCTEST}

[43] A separate approach to estimating the distribution of the maximum covariance involves searching for the maximum covariance in synthetic random records. Note that this approach, which we refer to as the Monte Carlo method, is distinct from the Monte Carlo multivariate integration method described in section 4 , which we refer to as the multivariate integration method. In cases of long time-uncertain series with large time uncertainties, it appears this approach is actually more efficient and we will have cause to use it in evaluating a record in the next section. In this Monte Carlo approach, we compute the distribution of the maximum covariance by generating random signals with the same distribution and the same autocovariance structure as $x(t)$. The degrees of freedom of the random synthetic series is thus the same as the time series to be tested and the test is no longer biased by the spurious covariance. To generate random synthetic series with the desired autocorrelation and distribution, we first form an autocovariance matrix with diagonal terms set to one and the $n$th off-diagonal terms set to the $n$th lag autocovariance. Next, let $L$ denote the Cholesky decomposition of the autocovariance matrix and $R$ denote a univariate normal random variable. Then the product $R L$ generates a random time series with the same expected autocovariance structure as $x(t)$ [e.g., Robinson, 2003]. The maximum covariance can then be sampled between a timeuncertain series and the randomly generated time series.
Repeating this procedure many times permits estimation of the distribution of the maximum covariance.

[44] Performing the search for the maximum covariance with each Monte Carlo realization involves adjusting the timescale and resampling the record, which results in minor changes in the mean and variance of each realization. This ability to influence the mean and variance of the time series through linear interpolation amounts to spurious sources of covariance which, unless controlled for, tend to result in maximum covariances that are slightly too high. We could increase the resolution of the data to minimize the influence of the linear interpolation step, but this increases the computational cost. Instead, we choose to renormalize each realization to have zero mean and unit standard deviation.

[45] Renormalizing each record effectively alters the distribution of maximum covariance, raising the question of whether this Monte Carlo procedure will yield results consistent with the analytical derivation. We note, however, that for a large sample size each realization has only small variations of mean and variance, so that the distribution resulting from the renormalized time series is very nearly unchanged. To demonstrate how renormalization affects the distribution, we computed the distribution of covariance between a fixed time series and the renormalized realizations of a normal random variable with different degrees of freedom. A quantile-quantile plot (see Figure 7) shows that renormalized series with ten degrees of freedom are not normally distributed, but when the random variable exceeds 40 degrees of freedom, the distribution appears effectively normal, indicating renormalization will then have little effect on the distribution.

[46] In the next section we demonstrate that the Monte Carlo method and the analytical method yield distributions of the maximum covariance that are consistent within the small uncertainty of the methods.

\section{Application to Dongge Cave}

[47] We can now apply our algorithm to actual climate data. We choose to test the maximum covariance of decadal variability between $\delta^{18} \mathrm{O}$ from Dongge cave, China [Wang et al., 2005], and the $\Delta^{14} \mathrm{C}$ data of Intcal98 [Stuiver et al., 1998]. This particular record is chosen for three reasons. First, in so much as correlation between these records does exist, it has important physical implications. Second, it is difficult to ascertain whether the records covary through visual inspection, thus recommending the use of a quantitative method. (Indeed, various scientists have suggested to us that visual inspection alone shows that the records are, or are not, covarying with one another.) Finally, in searching for a maximum covariance within the time uncertainty of the Dongge cave record, Wang et al. [2005] employed a method which directly parallels the assumptions made in developing the MCTEST, including assuming that the selected TCPs are independent and follow a uniform time distribution. The $\Delta^{14} \mathrm{C}$ record is assumed time certain. Note, however, that the time uncertainty of the Intcal98 $\Delta^{14} \mathrm{C}$ has elsewhere been modeled as normally distributed [Stuiver et al., 1998] and following a random walk [Buck and Blackwell, 2004]. 


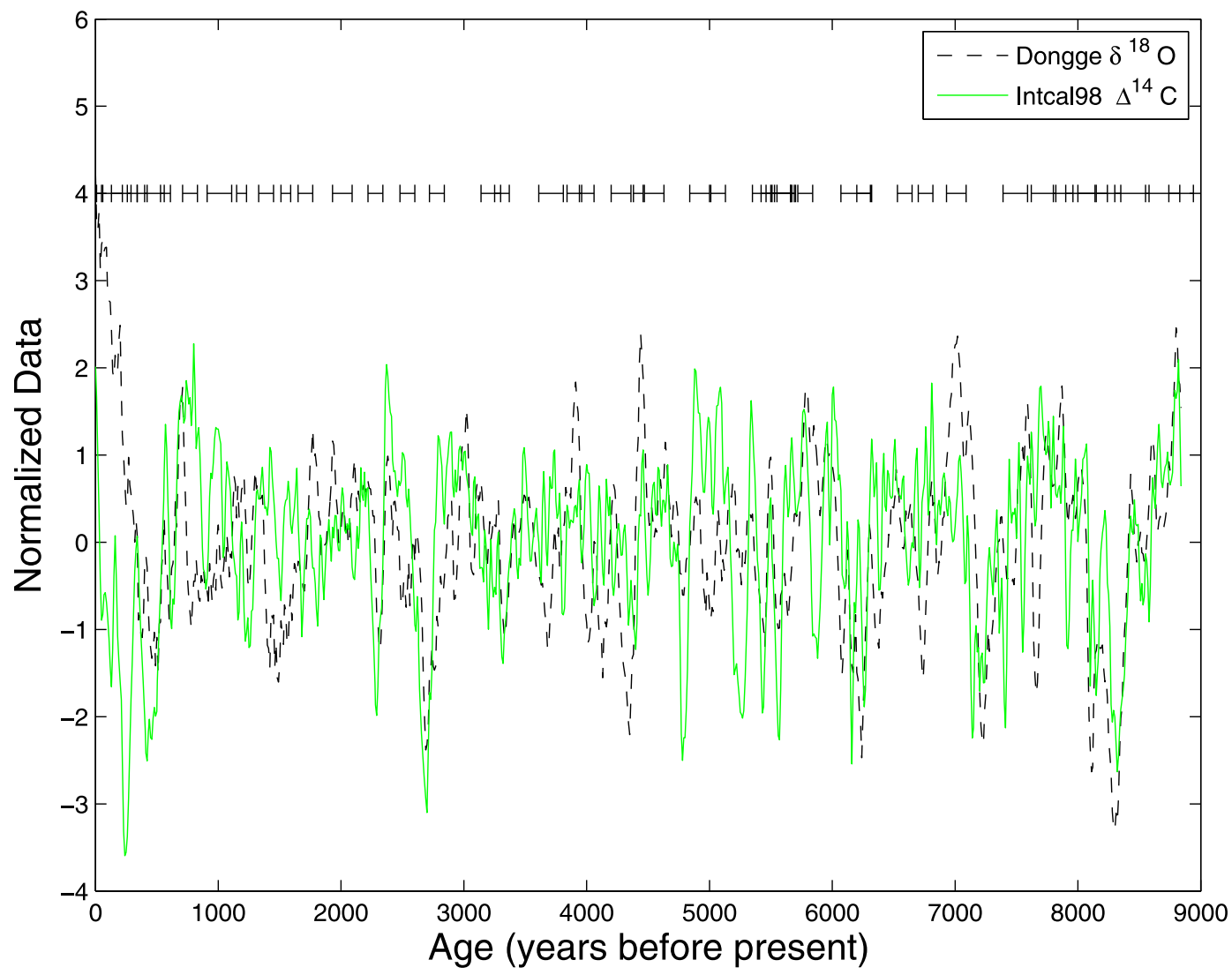

Figure 8. The time-uncertain $\delta^{18} \mathrm{O}$ series from Dongge Cave and the Intcal98 $\Delta^{14} \mathrm{C}$ record, which was tuned within the dating uncertainty to give a cross correlation of 0.3 . Both time series are normalized to zero mean and a standard deviation of one and are resampled at ten year intervals using linear interpolation. The horizontal bars indicate the time uncertainties associated with each radiometric date, which are used as time control points.

[48] Wang et al. [2005] obtained a maximum cross correlation of 0.3 between the $\delta^{18} \mathrm{O}$ and the $\Delta^{14} \mathrm{C}$ data by adjusting the time of the $\delta^{18} \mathrm{O}$ data within its radiometrically determined time uncertainties, and on this basis proposed that solar variability influences the Asian monsoons (see Figure 8). We seek to evaluate whether the maximum correlation coefficient of 0.3 is significant at the $95 \%$ confidence level.

[49] To preprocess the data in a manner similar to that of Wang et al. [2005], a third order high-pass Butterworth filter is applied to the $\delta^{18} \mathrm{O}$ speleothem record with a normalized cutoff frequency of $1 / 730$, selected to give the same maximum correlation of 0.3 reported by Wang et al. [2005]. We resample both the $\delta^{18} \mathrm{O}$ time-uncertain series from Dongge Cave and the $\Delta^{14} \mathrm{C}$ Intcal98 record at a 10 year resolution using linear interpolation and normalize to zero mean and unit variance. This results in 889 data points (the series are 8890 years long), with a decorrelation length of about 20 points or 200 years (see Figure 9). The degrees of freedom are, therefore, about 45 indicating that the mean and variances of the renormalized time series varies little and that the distribution of covariances between our renormalized realizations and the random variable is approximately normal.

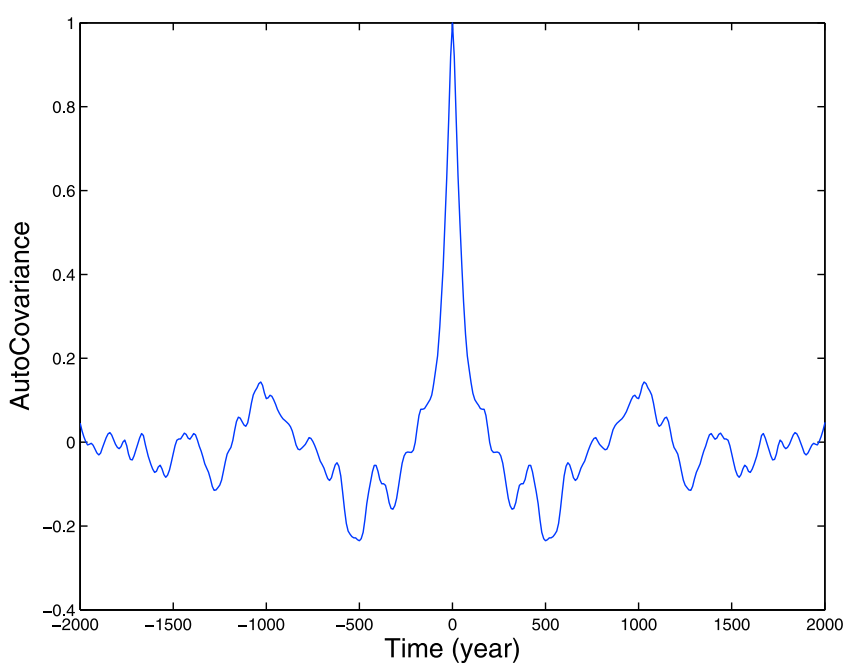

Figure 9. The sample autocovariance of the Intcal98 $\Delta^{14} \mathrm{C}$ record used to generate the random variable $X(t)$. The autocovariance approaches zero at 200 year lags. 


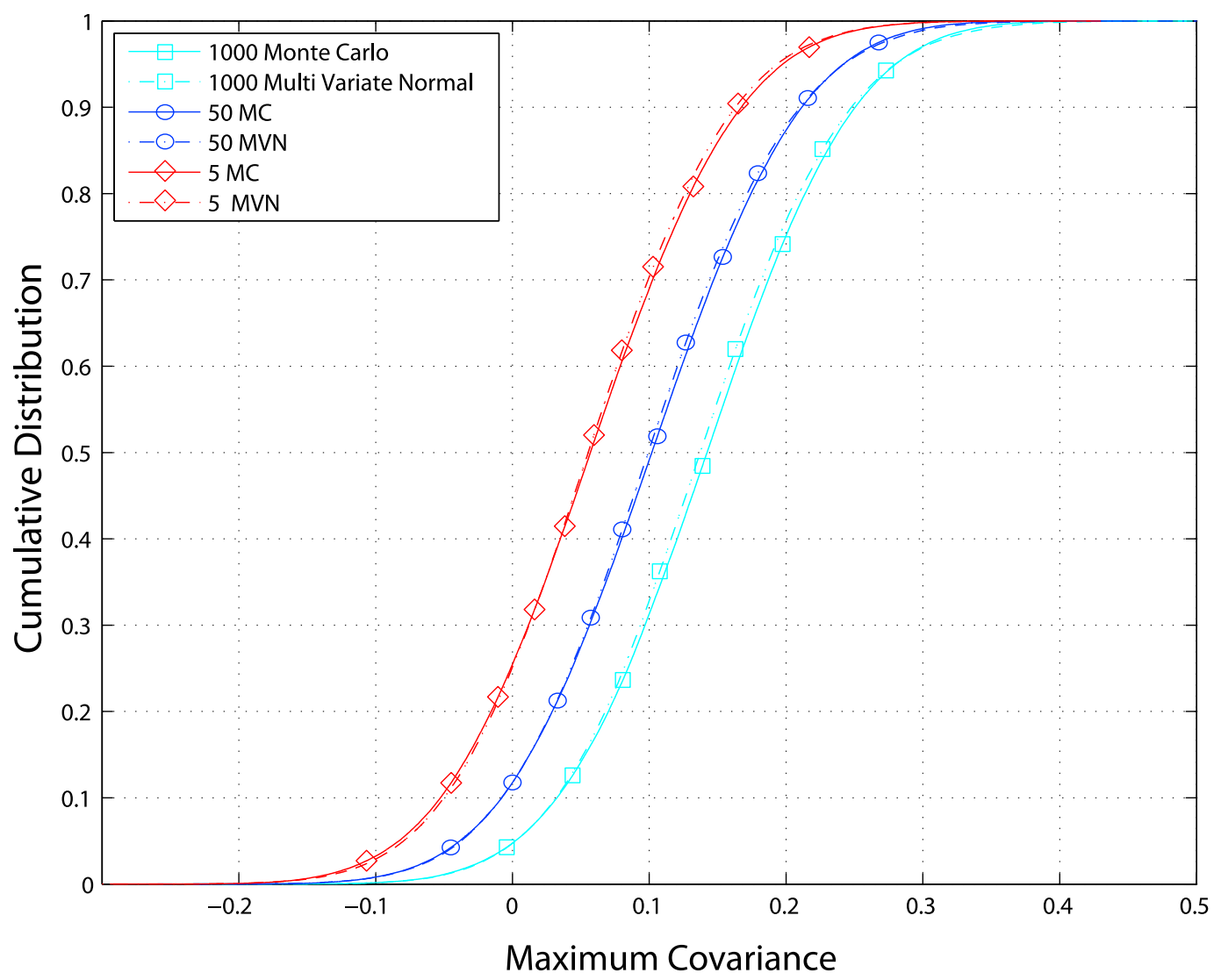

Figure 10. The cumulative distribution of the maximum covariance using Monte Carlo (MC) and multivariate normal integration (MVN) methods for sets of 1000,50 , and 5 realizations of time-uncertain $\delta^{18} \mathrm{O}$ series. The small offset is accounted for by the linear interpolation used to resample the data for each realization in the Monte Carlo method. As the number of realizations increases, the distribution shifts to the right and the $95 \%$ critical values increases.

[50] The $\Delta^{14} \mathrm{C}$ series is accepted as having fixed times, i.e., $x(t)$, and we only address the time uncertainties in the $\delta^{18} \mathrm{O}$ series from Dongge Cave, i.e., $y\left(n, t_{n}^{\prime}\right)$. The estimated time errors in the $\Delta^{14} \mathrm{C}$ series average 20 years, and these could be accounted for in the relative timing uncertainty, along with their autocorrelation structure, but we choose not to because the errors are relatively small and this would diverge from the method used by Wang et al. [2005]. Note that the autocovariance of $\delta^{18} \mathrm{O}$ approaches zero at lags of about 200 years. Thus, time adjustments of 200 years or larger would permit the $\Delta^{14} \mathrm{C}$ series to be compared against an essentially wholly new realization of $\delta^{18} \mathrm{O}$, acting to increase the maximum covariance associated with the null hypothesis and to decrease the power of the test. Fortunately, the Dongge cave data are exceptionally well dated and all time uncertainties are less than 200 years, averaging about 70 years.

[51] Forty-eight points of Dongge Cave data have radiometric time estimates and these are adopted as the TCPs. The uncertainties associated with these radiometric estimates are assumed to be discrete and uniformly distributed between the two sigma reported bounds on the time uncertainty. Given the 10 year resolution of the data, about $6 \times 10^{55}$ realizations of the series $y\left(n, t_{n}^{\prime}\right)$ are possible. However, since many of these realizations are highly correlated with each other, we selected the realizations that are maximally different from each other. That is, we only consider the time realized at the initial time estimate and the bracketing maximum and minimum change allowed. Even so, there are still too many $\left(>3^{40}\right)$ realizations to practically construct and evaluate the multivariate normal integral.

[52] In order to further decrease the dimension of the problem, we appeal to the asymptotic property shown in Figure 4, and randomly select some of the remaining realizations as follows. We let $S_{i}$ be a subset of realizations of $y\left(n, t_{n}^{\prime}\right)$ selected randomly from the set $S_{i+1}$. Since $S_{i+1}$ contains all possible $S_{i}$, the cumulative distribution of the maximum covariance $S_{i+1}$ must be greater than that of $S_{i}$. In particular, the $95 \%$ critical value of $S_{i+1}$ is higher than $S_{i}$. Thus, if the distribution derived from a multivariate normal integral using set $S_{i}$ fails to permit rejection of the null hypothesis, the distributions from all sets $S_{i+1}$ will necessarily also not permit rejection. To put it another way, the $p$ value for $S_{i+1}$ is always greater than or equal to the $p$ value of $S_{i}$. The tactic is to begin with a small set and test progressively larger subsamples of all possible realizations only as necessary.

[53] The distributions of the maximum covariance for each set was evaluated using both the Monte Carlo method and multivariate normal integration, yielding very similar results 


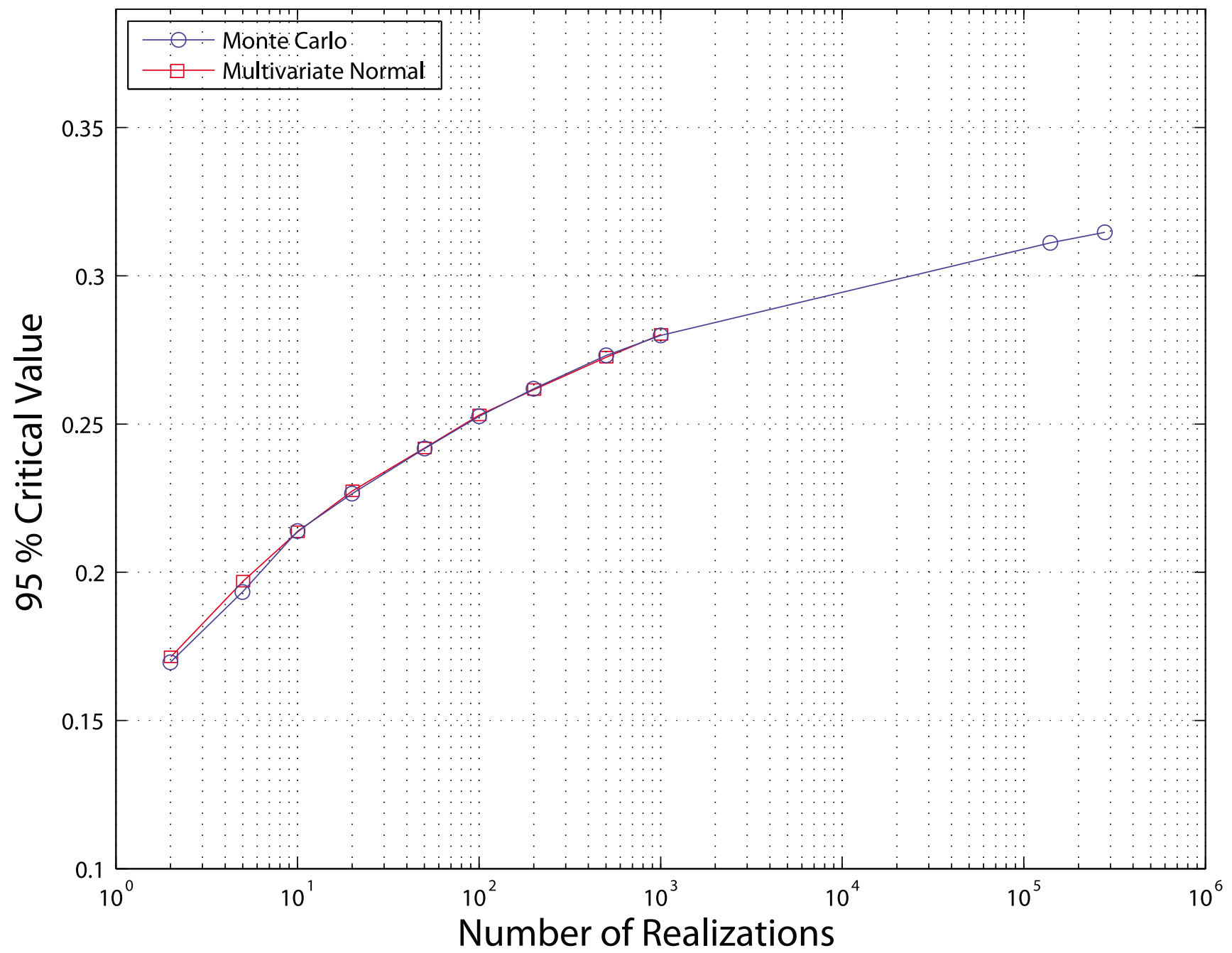

Figure 11. The $95 \%$ critical value for different size subsamples of the time-uncertain $\delta^{18} \mathrm{O}$ series from Dongge Cave, using both multivariate normal integral and Monte Carlo methods. The $95 \%$ critical value increases monotonically as the number of realizations increases. When the number of realizations is greater than 10,000, the multivariate normal integration method would require prohibitive computing resources and only the Monte Carlo method is used. For 140,000 realizations, the $95 \%$ critical value obtained from only the Monte Carlo method shows that a correlation of 0.3 is not significant at the $95 \%$ level. This correlation would appear even further from the $95 \%$ level were more realizations to be used in constructing the null hypothesis. The standard deviation of the offsets between the two methods tends to average about 0.007 , whereas the offsets shown here have a standard deviation of 0.002 .

(Figure 10). The small offset which does exist appears to owe to the linear interpolation issues associated with the Monte Carlo Method, discussed in the previous section. As the number of realizations in the set $S_{i}$ increases, the resulting distribution of the null hypothesis shifts toward larger covariance values. As noted, the Monte Carlo approach is computationally more efficient than the multivariate integration approach when dealing with large numbers of time realizations, and only the Monte Carlo procedure is utilized for covariance matrices containing more than 10,000 realizations.

[54] Figure 11 shows the $95 \%$ critical value obtained from both the Monte Carlo approach and analytical methods for different sizes of realizations. When the number of realiza- tions becomes 140,000 , the $95 \%$ critical value obtained from the Monte Carlo method indicates that a correlation of 0.3 is insignificant at the $95 \%$ level. We are therefore unable to reject the null hypothesis of no covariance between the Dongge Cave $\delta^{18} \mathrm{O}$ and atmospheric $\Delta^{14} \mathrm{C}$. Note that we have only considered a small subset of possible time model adjustments $\left(140,000 / 10^{55} \sim 0\right)$. Inclusion of more realizations could only strengthen the conclusion that there is insignificant evidence for covariance between these climate variables. Note, however, that we have only tested a linear, zero-lag covariance between the $\delta^{18} \mathrm{O}$ from Dongge Cave and the $\Delta^{14} \mathrm{C}$ from Intcal98 the possibility of a lead/lag

\footnotetext{
${ }^{1}$ Auxiliary materials are available in the HTML. doi:10.1029/ 2008PA001713.
} 
relationship or some nonlinear relationship has not been explored.

\section{Extensions and Conclusion}

[55] The MCTEST permits assessment of the statistical significance of the Maximum Covariance between timeuncertain series. We have sought to provide a simple and practical derivation as well as an application of this method. There are, however, many directions in which the test can be further developed.

[56] Covariance is used as a measure of similarity, and thus the test is most directly applied to linear, in-phase relationships between stationary time series. If one seeks to test or admit the possibility of leads and lags in timeuncertain series, it may be useful to introduce a time offset parameter, permitting for shifting the times across the entire time-uncertain data series, in addition to the more local shifts in time permitted by the uncertain TCPs. It may also be useful to apply different measures between the time series for testing for the presence of nonlinear relationships or covariance between nonstationary data, e.g., the cointegration statistic [Granger, 1981].

[57] The time uncertainty associated with each TCP has been assumed to follow a uniform distribution. It may be possible to extend the test to a nonuniform distribution, accounting for the varying probability of each realization. Consideration of correlation between TCP variations, for example, because of systematic errors associated with radiometric dates, would also be of use.

[58] It has also been assumed that time uncertainties take on discrete values. In principle, acceptable times are continuous, but this would generate an infinite number of realizations. We chose to select realizations that are far apart from one another to efficiently evaluate the distribution of the maximum covariance. Depending on interest and computational resources, an efficient optimization algorithm such as Dynamic Time Warping [e.g., Sakoe and Chiba, 1990; Tomasi et al., 2004] can be employed to search for the maximum covariance out of large number of possible realizations.

[59] Finally, we note that while we have assumed that the climate data are normally distributed, in practice any distribution could be utilized with the Monte Carlo method we propose. For example, univariate random numbers with bimodal distribution can be multiplied by the Cholesky decomposition of the covariance matrix to generate multivariate bimodal random variables. An alternate method is to directly generate surrogate time series having a similar autocorrelation and distribution as one of the time-uncertain series [Schreiber and Schmitz, 2000]. It may also be possible to employ a nonnormal multivariate integral to analytically derive the distribution of maximum covariance.

[60] In conclusion, the MCTEST presented here permits for statistical testing of the covariance between time-uncertain records and, thus, is applicable to the evaluation of potential interrelationships between many paleoclimate and geologic data series. Extensions of the MCTEST can readily be made, which should serve to widen its applicability, and these will be taken up in subsequent work. We hope that this test will provide for more rigorous assessment of paleoclimate observations and thereby further the understanding of climate. Software for conducting the MCTEST is available in the auxiliary material. ${ }^{1}$

[61] Acknowledgments. We are grateful for comments by Carl Wunsch, Martin Tingley, Ethan Butler, Nathan Arnold, and Andrew Rhines, as well as for comments by two anonymous reviewers. Funding was provided by the National Science Foundation, award OCE-0645936.

\section{References}

Aach, J., and G. Church (2001), Aligning gene expression time series with time warping algorithms, Bioinformatics, 17(6), 495-508.

Afonja, B. (1972), The moments of the maximum of correlated normal and t-variates, $J . R$. Stat. Soc., Ser. B, 34, 251-262.

Anderson, E., et al. (Eds.) (1999), LAPACK User's Guide, 3rd ed., Soc. for Ind. and Appl. Math., Philadelphia, Pa.

Anstey, N. (1964), Correlation techniques: A review, Geophys. Prospect., 12(4), 355-382.

Barnhart, C., and K. T. Talluri (1997), Airlines operations research, in Design and Operation of Civil and Environmental Engineering Systems, 1st ed., edited by C. ReVelle and A. E. McGarity, pp. 435-469, John Wiley, New York.

Blaauw, M., B. Van Geel, D. Mauquoy, and J. Van Der Plicht (2004), Carbon-14 wigglematch dating of peat deposits: Advantages and limitations, J. Quat. Sci., 19(2), 177-181.

Buck, C., and P. Blackwell (2004), Formal statistical models for estimating radiocarbon calibration curves, Radiocarbon, 46(3), 10931102.

Casella, G., and R. L. Berger (Eds.) (2002), Statistical Inference, 2nd ed., Duxbury, Pacific Grove, Calif.

Clark, P. U., S. W. Hostetler, N. G. Pisias, A. Schmittner, and K. J. Meissner (2007),
Mechanisms for an $\sim 7-\mathrm{kyr}$ climate and sealevel oscillation during marine isotope stage 3 , in Ocean Circulation: Mechanisms and Impacts, Geophys. Monogr. Ser., vol. 173, edited by A. Schmittner, J. Chiang, and S. Hemmings, pp. 209-246, AGU, Washington, D. C.

Clark, R. (1989), A randomization test for the comparison of ordered sequences, Math. Geol., 21(4), 429-442.

Clark, R., and R. Thompson (1979), A new statistical approach to the alignment of time series, Geophys. J. R. Astron. Soc., 58, 593-607.

Clemens, S. (2005), Millennial-band climate spectrum resolved and linked to centennial-scale solar cycles, Quat. Sci. Rev., 24, 521-531.

Comet, J. P., J. C. Aude, E. Glemet, J. L. Risler, A. Henaut, P. P. Slonimski, and J. J. Codani (1999), Significance of Z-value statistics of Smith-Waterman scores for protein alignments, Comp. Chem., 23(3-4), 317-331.

Crowley, T. (1999), Correlating high-frequency climate variations, Paleoceanography, 14(3), $271-272$.

David, F. (Ed.) (1938), Tables for the Correlation Coefficient, Cambridge Univ. Press, Cambridge, U. K.

David, F. (1953), A note on the evaluation of the multivariate normal integral, Biometrika, 40, $458-459$.
David, H. A., and H. N. Nagaraja (Eds.) (2003), Order Statistics, 3rd ed., John Wiley, Hoboken, N. J.

Delmotte, M., J. Chappellaz, E. Brook, P. Yiou, J. M. Barnola, C. Goujon, D. Raynaud, and V. I. Lipenkov (2004), Atmospheric methane during the last four glacial-interglacial cycles: Rapid changes and their link with Antarctic temperature, J. Geophys. Res., 109, D12104, doi:10.1029/2003JD004417.

Genty, D., D. Blamart, R. Ouahdi, M. Gilmour, A. Baker, J. Jouzel, and S. Van-Exter (2003) Precise dating of Dansgaard-Oeschger climate oscillations in western Europe from stalagmite data, Nature, 421, 833-837.

Genz, A. (1993), Comparison of methods for the computation of multivariate normal probabilities, Comput. Sci. Stat., 25, 400-405.

Gordon, A., and S. Buckland (1996), A permutation test for assessing the similarity of ordered sequences, Math. Geol., 28(6), 735-742.

Granger, C. W. J. (1981), Some properties of time series data and their use in econometric model specification, J. Econometrics, 16, $121-130$.

Granger, C. W. J., and P. Newbold (1974), Spurious regressions in econometrics, $J$. Econometrics, 2(2), $111-120$. 
Gupta, S. S. (1963), Probability integrals of multivariate normal and multivariate $t$, Ann. Math. Stat., 34, 792-828.

Hendy, I. L., J. P. Kennett, E. B. Roark, and B. L. Ingram (2002), Apparent synchroneity of submillennial scale climate events between Greenland and Santa Barbara Basin, California from 30-10 ka, Quat. Sci. Rev., 21, 1167-1184.

Huang, X., A. Acero, and H.-W. Hon (Eds.) (2001), Spoken Language Processing, 1st ed., Prentice, Hall, Uer Saddle River, N. J.

Husler, J. (1961), A formula for the tail probability of a multivariate normal distribution and its applications, Oper. Res., 9, 145-162.

Huybers, P., and C. Wunsch (2004), A depthderived Pleistocene age model: Uncertainty estimates, sedimentation variability, and nonlinear climate change, Paleoceanography, 19, PA1028, doi:10.1029/2002PA000857.

Karlin, S., and S. F. Altschul (1990), Methods for assessing the statistical significance of molecular sequence features by using general scoring schemes, Proc. Natl. Acad. Sci. U. S. A., 87(6), 2264-2268.

Kotz, S., (Ed.) (2000), Extreme Value Distributions, 1st ed., Imp. College Press, London.

Levitt, M., and M. Gerstein (1998), A unified statistical framework for sequence comparison and structure comparison, Proc. Natl. Acad. Sci. U. S. A., 95(11), 5913-5920.

Lisiecki, L. E., and P. A. Lisiecki (2002), Application of dynamic programming to the correlation of paleoclimate records, Paleoceanography, 17(4), 1049, doi:10.1029/ 2001PA000733.

Little, M. G., R. R. Schneider, D. Kroon, B. Price, T. Bickert, and G. Wefer (1997), Rapid palaeoceanographic changes in the Benguela upwelling system for the last 160,000 years as indicated by abundances of planktonic foraminifera, Palaeogeogr. Palaeoclimatol. Palaeoecol., 130, 135-161.

Macleod, N., and P. Sadler (1995), Estimating the line of correlation, in Graphic Correlation, edited by K. O. Mann and H. R. Lane, Spec. Publ. SEPM Soc. Sediment. Geol., 53, 51-64.

Moran, P. A. P. (Ed.) (1968), An Introduction to Probability Theory, 1st ed., Clarendon, Oxford, U. K.
Mudelsee, M. (2001), The phase relations among atmospheric $\mathrm{CO}_{2}$ content, temperature and global ice volume over the past $420 \mathrm{ka}$, Quat. Sci. Rev., 20(4), 583-589.

Oppo, D. W., B. K. Linsley, Y. Rosenthal, S. Dannenmann, and L. Beaufort (2003), Orbital and suborbital climate variability in the Sulu Sea, western tropical Pacific, Geochem Geophys. Geosyst., 4(1), 1003, doi:10.1029/ 2001GC000260.

Pahnke, K., R. Zahn, H. Elderfield, and M. Schulz (2003), 340,000-year centennialscale marine record of Southern Hemisphere climate oscillation, Science, 301, 948-952.

Peterson, L. C., G. H. Haug, K. A. Hughen, and U. Röhl (2000), Rapid changes in the hydrologic cycle of the tropical Atlantic during the last glacial, Science, 290(5498), 1947-1951.

Rahmstorf, S. (2003), Timing of abrupt climate change: A precise clock, Geophys. Res. Lett. 30(10), 1510, doi:10.1029/2003GL017115.

Robinson, P. M. (Ed.) (2003), Time Series With Long Memory, 1st ed., Oxford Univ. Press, Oxford, U. K.

Sadler, P. M. (1981), Sediment accumulation rates and the completeness of stratigraphic sections, J. Geol., 89, 569-584.

Sakoe, H., and S. Chiba (1990), Dynamic programming algorithm optimization for spoken word recognition, in Readings in Speech Recognition, edited by A. Waibel and K.-F. Lee, pp. 159-165, Morgan Kaufmann, San Mateo, Calif.

Schreiber, T., and A. Schmitz (2000), Surrogate time series, Physica D, 142, 346-382.

Schulz, H., U. Rad, and H. Erlenkeuser (1998) Correlation between Arabian Sea and Greenland climate oscillations of the past 110,000 years, Nature, 393, 54-57.

Shaw, A. B. (1964), Time in Stratigraphy, McGraw-Hill, New York

Sheppard, W. F. (1897), On the geometrical treatment of the "normal curve" of statistics, with especial reference to correlation and to the theory of error, Proc. R. Soc. London, 62, $170-173$.

Solé, J., A. Turiel, and J. E. Llebot (2007), Using empirical mode decomposition to correlate pa- leoclimate time-series, Nat Hazards Earth Syst. Sci., 7, 299-307.

Stott, L., C. Poulsen, S. Lund, and R. Thunell (2002), Super ENSO and global climate oscillations at millennial time scales, Science, 297. $222-226$.

Stuiver, M., et al. (1998), INTCAL98 radiocarbon age calibration, 24,000-0 cal BP Radiocarbon, 40, 1041-1083.

Tomasi, G., F. van den Berg, and C. Andersson (2004), Correlation optimized warping and dynamic time warping as preprocessing methods for chromatographic data, J. Chemometrics, $18,231-241$.

Tong, Y. (Ed.) (1990), The Multivariate Normal Distribution, 1st ed., Springer, Atlanta, Ga.

Wang, Y. J., H. Cheng, R. L. Edwards, Z. S. An

J. Y. Wu, C.-C. Shen, and J. A. Dorale (2001), A high-resolution absolute-dated late Pleistocene monsoon record from Hulu Cave, China, Science, 294, 2345-2348.

Wang, Y., et al. (2005), The Holocene Asian monsoon: Links to solar changes and North Atlantic climate, Science, 308(5723), 854-857.

Wunsch, C. (2003), Greenland-Antarctic phase relations and millennial time-scale climate fluctuations in the Greenland cores, Quat. Sci. Rev., 22, 1631-1646.

Wunsch, C. (2006), Abrupt climate change: An alternative view, Quat. Res., 65, 191-203.

Wunsch, C. (2008), Extremes, patterns, and other structures in oceanographic and climate records, paper presented at Aha Hulikoa Hawaiian Winter Workshop on Extreme Events, U.S. Off. of Nav. Res., Honolulu, Hawaii.

Yule, G. U. (1926), Why do we sometimes get nonsense-correlations between time-series?A study in sampling and the nature of timeseries, J. R. Stat. Soc., 89, 1-63.

E. Haam, Applied Mathematics, Harvard University, Cambridge, MA 02138, USA. (keh@eecs. harvard.edu)

P. Huybers, Department of Earth and Planetary Sciences, Harvard University, Cambridge, MA 02138, USA 